\title{
Clinical and Demographic Profile of Patients Receiving ECT in an Institute
}

\author{
Sherchan $\mathrm{S}^{1}$, J oshi $\mathrm{D}^{1}$ \\ ${ }^{1}$ Psychiatrist, Mental Hospital, Lalitpur, Nepal
}

\begin{abstract}
Background: Despite being an effective and safe treatment for severe psychiatric disorders, the use of electroconvulsive therapy (ECT) in different countries and different institutions has been uneven. More-over, in developed countries its use has clearly shown a declining trend.

Methods: This retrospective study using ECT case record book has sought to find out the clinical and demographic profile of patients receiving ECT at the Mental Hospital in Lagankhel, Lalitpur and also to determine the trend of its use in last 10 years. Two ECT case record books which covered the time period from 1999 to 2008 were studied. Retrospective data necessary for this study was collected from those records.

Results: The total number of ECT received in the year 1999 was 902 while it was 242 in the year 2008. The number of patients receiving ECT in the year 2008 was 44 patients with the diagnosis of acute psychosis and schizophrenia while only 20 patients with the diagnosis of affective disorder (mania and depression) out of total 64 patients.

Conclusion: The study showed declining trend of the use of ECT in the state Mental Hospital as in developed countries but in contrast to affective disorder, acute psychosis and schizophrenia were the predominant clinical conditions where ECT was used.
\end{abstract}

Key words: Direct/Unmodified, Electroconvulsive Therapy, Psychiatric disorders, Psychotropic medications

\section{INTRODUCTION}

Electroconvulsive therapy is an effective and relatively safe treatment for severe psychiatric disorders, including severe depressive disorders, mania, catatonia and acute exacerbations of schizophrenia. ${ }^{1}$ With the introduction of effective psychotropic medications in the 1950s, use of ECT has declined in the developed countries. ${ }^{2,3}$ However,
ECT as a therapeutic tool is probably used more widely in developing countries like Nepal, though formal data on this matter is lacking.

Very less number of psychiatrists, less availability of treatment facilities in comparison to the population in need, necessity of cutting down the costs of treatment because of poor socioeconomic status of the patients,

Correspondence: Dr. Surendra Sherchan, Mental Hospital, Lalitpur, Nepal, Phone: 9841904228, Email: drsherchan_s@yahoo.com 
may be some factors associated with greater use of ECT in developing countries. This article has tried to explore the clinical and demographic profiles of the patients receiving ECT at the only state owned mental hospital in Lalitpur. In addition, it has also tried to look at the trends in the number of ECT being provided in the last ten years at the same center.

\section{METHODS}

A retrospective descriptive study using data recorded in the ECT record book of the Mental hospital, Lalitpur was carried out. Total number of ECT provided annually from 1999 AD to 2008 AD was calculated and the trend was analyzed.

Clinical and demographic profiles of the patients receiving $E C T$ in $2008 A D$, was analyzed for total number of patients, total number of ECT given, number of ECT per patient and the clinical indications for which ECT was used.

All patients received direct / unmodified ECT. Injection Atropine $0.6 \mathrm{mg}$ given intramuscularly, half an hour before the ECT, was the only pre-medication used. Data entry and statistical analysis were done in Microsoft Office Excel 2007 and Statistical Package for Social Sciences (SPSS) 13.0. Ethical approval for the study was obtained from the hospital research committee and confidentiality of the patients and their data was strictly maintained.

\section{RESULT}

A total of 64 patients received ECT in 2008. Among the total patients, 42 were males and 22 were females. All of the patients were inpatients. No ECT service was provided on an outpatient basis.

\section{Table 1. Distribution of patients by age}

\begin{tabular}{|lc|}
\hline Age Range (in years) & Number of patients \\
\hline $10-19$ & 13 \\
$20-29$ & 28 \\
$30-39$ & 18 \\
$40-49$ & 3 \\
$50-59$ & 1 \\
$60-69$ & 1 \\
Total & 64 \\
\hline \hline
\end{tabular}

The age range of the patients was from 16 to 65 years. Most commonly, patients within the age range of 20 to 40 years received ECT treatment.
Table 2. Clinical diagnoses of the patients receiving ECT

\begin{tabular}{|c|c|}
\hline Diagnoses & $\begin{array}{c}\text { Number of } \\
\text { patients }\end{array}$ \\
\hline Acute and Transient Psychotic disorder & 19 \\
\hline Schizophrenia (Catatonic) & 1 \\
\hline Schizophrenia (Paranoid) & 1 \\
\hline $\begin{array}{l}\text { Schizophrenia (Unclassified / Not } \\
\text { categorized) }\end{array}$ & 18 \\
\hline $\begin{array}{l}\text { Psychosis Not Otherwise Specified } \\
\text { (including Post Partum Psychosis) }\end{array}$ & 5 \\
\hline Bipolar affective disorder / Mania & 15 \\
\hline Depression & 5 \\
\hline Total & 64 \\
\hline
\end{tabular}

Among the patients receiving ECT, acute and transient psychotic disorder was the commonest diagnosis, followed by Schizophrenia. Depressive disorders were surprisingly less common.

\begin{tabular}{|cc|}
\hline Table 3. Number of ECT treatments per patient \\
\hline Number of ECT treatment & Number of patients \\
\hline 1 & 1 \\
2 & 4 \\
3 & 4 \\
4 & 16 \\
5 & 12 \\
6 & 24 \\
7 & 2 \\
8 & 1 \\
\hline Total & 64 \\
\hline
\end{tabular}

Maximum number of patients received four to six cycles of ECT. A few patients, especially those with catatonic symptoms received just one to two cycles of ECT. Patients with Mania, in whom the aim of the treatment was just to control the aggression and over activity, were also among those who received less than four cycles of ECT.

There was no major complication seen after the use of ECT. There were three cases with minor complications like lip bite. All other cases were uneventful with no physical complications. Cognitive outcomes following ECT treatment was however not assessed in this study.

The total number of ECT being given each year has been declining significantly over the last decade. When compared to $1999 \mathrm{AD}$, the number of ECT treatments per year has decreased by more than $50 \%$ in the last few years. 


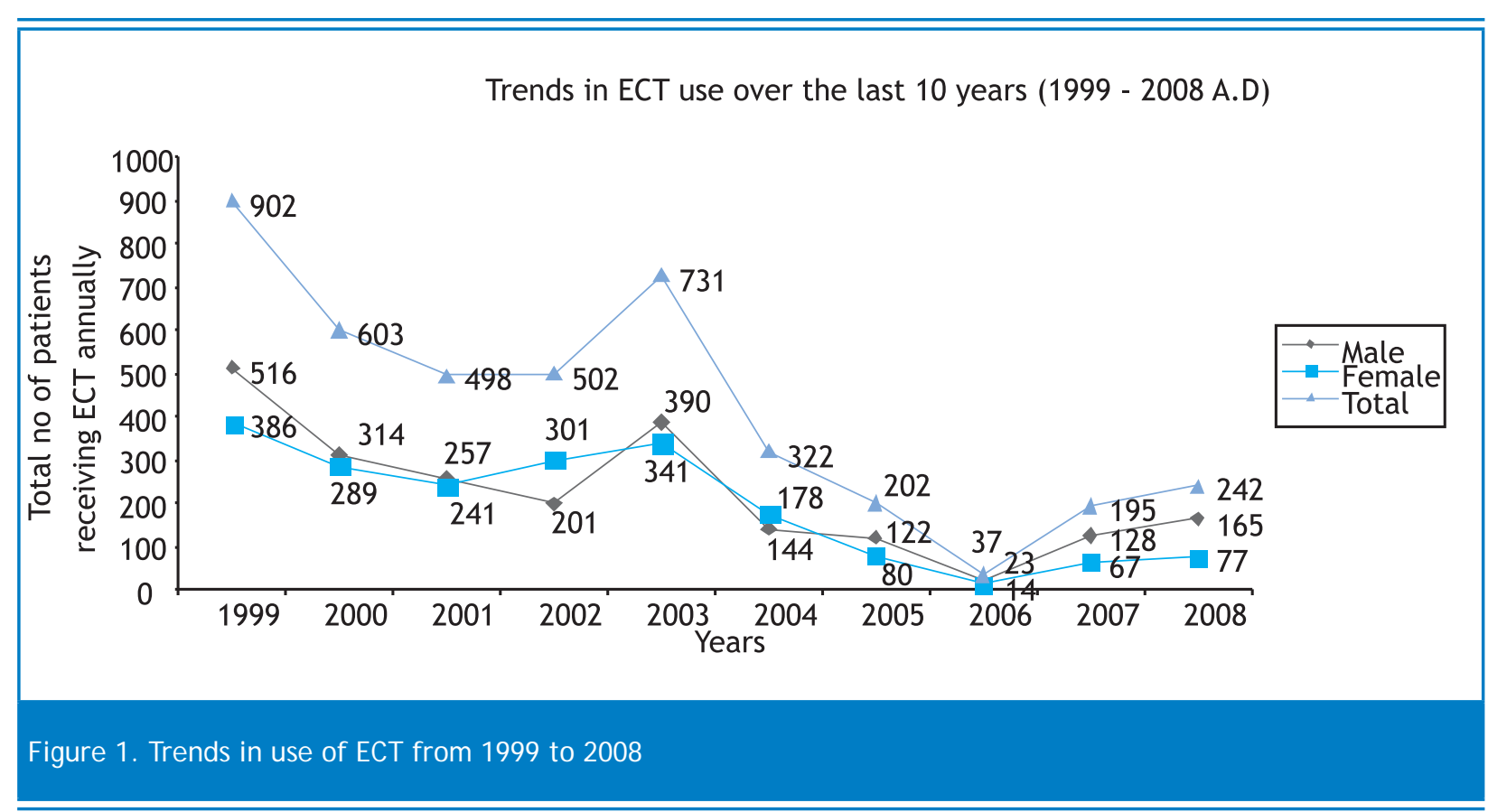

\section{DISCUSSION}

The trend in the number of ECT treatments per year has shown a constantly declining trend. When compared to the number of ECT treatments given in 1999 AD, the numbers have considerably decreased by more than $50 \%$ in the last few years. This is comparable to the trend in the developed countries. ${ }^{2}$ This decline may be due to the availability of newer psychotropic agents which has lead to a decrease in the need for ECT treatments.

Maj ority of the patients who received ECT treatments were diagnosed as having acute and transient psychotic disorder, followed by schizophrenia, whereas the number of patients with a diagnosis of depression was surprisingly less. This is in contrast to the data from other countries, where among the patients receiving ECT, the most common diagnosis is affective disorder, especially depressive disorder. ${ }^{3-8}$ Studies have shown that more than 80 percent of the patients receiving ECT in the United States are diagnosed as having affective disorders. ${ }^{2}$ The number of patients receiving ECT with a diagnosis of other disorders, including Schizophrenia has been declining since the 1970s. ${ }^{2}$ This discrepancy in the current study may be due to more numbers of acute psychoses and schizophrenia cases being admitted in the hospital

The typical number of ECT treatments varies by the clinical diagnosis and patient response. Standard texts recommend courses of five to ten treatments for acute disorders, although a larger number may be required for refractory conditions. ${ }^{7}$ In this study, regarding the number of ECT treatments, on an average, the majority of the patients have received four to six cycles of treatment, which is in par with the data from other countries. Lesser number of treatments that is just one to two cycles was used for patients with features of catatonia or to control agitation and violence in acute psychosis and mania.

ECT is used for very similar indications in Nepal as in the developed countries with very similar results; however, the method of administering ECT in centers like the Mental Hospital is very different. Though it is the only government owned center for providing specialized psychiatric services, almost all of the ECT recipients receive direct / unmodified ECT. This is due to various reasons including poor infrastructure, lack of anesthetic services, lack of other staffs for providing modified ECT and financial constraints. Though many studies have shown that unmodified ECT leads to more physical morbidities than the modified ECT few Indian studies have reported somewhat different findings. ${ }^{4}$ They have found that the incidence of complications in unmodified ECT was less than $1 \%$ if the treatment was provided by a skilled person after taking necessary precautions. ${ }^{5}$ Whereas, in many patients receiving modified ECT, there was more risk of potentially fatal complications like cardio-respiratory arrest, secondary to the use of anesthetic agents. ${ }^{6}$ In the current study also it was found that there were no significant complications during or after the ECT treatments. Thus in poor resource settings like ours, the administration of unmodified ECT by trained manpower, in the absence of anesthetic services, may be justified. However, this does not negate the need to start modified ECT services at the center in the days to come. 
The maximum number of patients receiving ECT treatments was within the age range of 20 to 40 years. This corroborates with the fact that majority of the psychiatric morbidities, including depression; bipolar disorder and schizophrenia have an onset in this age group. ECT was very infrequently used in the extremes of ages, and that too only when it was very necessary, in order to avoid complications of unmodified ECT in these age groups.

The data shows that ECT was administered only to the inpatients admitted at the hospital, however, if need arises, starting OPD based regular and maintenance ECT services can also be planned in future in order to reduce the costs and duration of hospital stay, keeping the poor resources, limited number of beds for admission and the need to cater to an increasing number of patients, in view.

Due to the unavailability of other similar studies in Nepal, the results couldn't be compared with other national data. Hence, whether the declining trend in the number of ECT administered, which was observed at the mental hospital, was the trend throughout the country or not couldn't be ascertained. Though ECT is known to be associated with a range of cognitive side effects, the current study has focused only on the physical complications following ECT. Moreover, patient outcomes and effects on the total duration of hospital stay were also not assessed.

\section{CONCLUSION}

Our study found out that the use of ECT as a therapy is decreasing over the years. Patients aged 20 to 40 received the highest number of ECTs and Acute and transient psychotic disorder was the most common indication for ECT. ECT was found to be relatively safe with only three cases of complications that included lip bites.
ECT has better efficacy, is more cost effective and safer. So despite a declining trend for its use in routine therapeutic practice, it would be logical to recommend its wider use in both in and outpatient settings with application of modern guidelines and methods for emergency need of treatment for severe psychiatric disorders. A multi centric study in tertiary care psychiatric centers in the country is needed to ascertain if our findings represent the general national trend.

\section{REFERENCE}

1. American PsychiatricAssociation. The practice of electroconvulsive therapy: recommendations for treatment, training, privileging. Washington DC: American Psychiatric Association; 1990.

2. Rosenbach ML, Hermann RC, Dorwart RA. Use of electroconvulsive therapy in the medicare population between 1987 and 1992. Psychiatr Serv 2000;48(12):1537-42.

3. Sylvester AP, Mulsant BH, Chengappa KN, Sandman AR, Haskett RF. Use of electroconvulsive therapy in a state hospital: a 10 year review. J Clin Psychiatry 2000;61(7):534-9.

4. Andrade C, Agarwal AK, Reddy MV. The practice of ECT in India: the practical administration of ECT. Indian J Psychiatry 1993;35(2):81-6.

5. Tharyan P, Saju JP, Dutta S, John KJ, Kuruvilla K. Physical morbidity with unmodified ECT: a decade of experience. Indian J Psychiatry 1993;35(4):211-4.

6. Trivedi JK, Mahendru S. ECT practices in India - special considerations. Arch Indian Psychiatry 2000;6(1):123-6.

7. Shapira B, Calev A, Lerer B. Optimal use of electroconvulsive therapy: choosing a treatment schedule. Psychiatr Clin North Am 1991;14:935-6.

8. Thompson JW, Weiner RD, Myers CP. Use of ECT in the United States in 1975, 1980 and 1986. Am J Psychiatry 1994;151:165761 